\title{
Effective high-quality science graphics from s-Ink.org
}

\author{
Fabio Crameri ${ }^{1,2}$, Grace E. Shephard ${ }^{2}$, and Eivind O. Straume ${ }^{2,3}$ \\ ${ }^{\mathrm{I}}$ Undertone.design, Bern, Switzerland \\ ${ }^{2}$ Centre for Earth Evolution and Dynamics, University of Oslo, Norway \\ ${ }^{3}$ The Jackson School of Geosciences, University of Texas at Austin, USA \\ Correspondence: Fabio Crameri (www.fabiocrameri.ch)
}

Non-peer reviewed preprint submitted to EarthArXiv

February 15, 2022

Crameri, F., G.E. Shephard, and E.O. Straume (2022, Pre-print), Effective high-quality science graphics from s-Ink.org, EarthArXiv

\begin{abstract}
standard internet image search on a scientific topic is common practice, and offers a plethora of images. However, the suggested images provide neither a guarantee in the accuracy of the science content being portrayed, nor in their clarity or artistic elegance. Being curated by both scientists and artists, the online s-Ink collection (https://s-Ink.org) is, in contrast, an exchange platform for high quality, science-related graphical products. The collection is highly structured and effectively searchable via text, keyword, or category. As necessary, its hosted and linked content can be regularly updated to maintain pace with advances in both scientific understanding and graphicrelated techniques. Key related information is provided alongside the graphical products, including an explanatory caption, the given license guiding further use, the names of their creators to value their work, and any related publication for proper citing and further in-depth information. By being open for contributions, the s-Ink collection facilitates access to open-access, community-driven, visually engaging, and science-proof graphics for free, while honouring the work that went into them.
\end{abstract}

\section{Introduction}

From presentations to papers, and from meetings to public outreach, digital artwork provides an extremely effective means to communicate scientific features and processes (e.g., Illingworth, 2017). This can include simple static images, to animated GIFs, to full cinematic-style movies. However, creating effective graphics takes a long time and requires both a reasonable knowledge of the processes and dynamics that are to be portrayed, and graphical skills for them to be effective and visually appealing (thus encouraging their reuse). An image search (e.g. 'mantle convection' or 'hydrological cycle') on the internet reveals a multitude of (geo)scientific artwork in existence. Unfortunately, however, many of the available artworks are produced by artists that may have have left out, or misrepresented, crucial details, or have been produced by scientists that failed to produce an eye-pleasing product (or perhaps even incorporate dated ideas or widely-inaccessible graphical aspects). 


\section{Shortcomings of common science graph- ics}

The image quality in a scientific (i.e., not artistic) sense can easily be checked. Critical image pitfalls are counted for relevant key Earth Science topics on one of the most common science-images retrieval platforms (see Figure A.I) and the results (as of December 202I) presented in Figure I. These are data misrepresentation (Figure Ia), bred by missing or faulty scales (e.g., colour bars with perceptually uneven colour gradients) obstructing the factual representation of data gradients, visual inaccessibility (Figure $\mathrm{Ib}$ ), provoked by unintuitive colour orders or colour combinations excluding colourvision deficient readers (e.g., green and red combinations), and conceptual misrepresentation (Figure Ic), brought about by outdated and wrong scientific content such as the likes of a "molten mantle" or the "Ist order geodynamic connection between mantle plumes and spreading ridges". The presence of those critical image pitfalls in a commonly accessible science image resource is striking. Universally accessible science graphics free of data misrepresentation and/or misleading concepts are rare. Furthermore, many images are available in low-resolution only, might not be suitable to variable backgrounds, and have limited or unclear copyright usage purposes. Conversely, a situation may arise in which someone has produced an image they are keen to share more widely but don't know what channels are available to increase its visibility.

In summary: open-accessible scientific-style artwork that looks nice, but also represents scientific and, in particular, geoscientific content clearly and accurately is uncommon and hard to find. It is in the interests of all scientists to share their research in an accessible, informative, aesthetic, and reproducible way (Makri, 2017); and this includes stand-alone graphical products.

\section{Graphics categories}

A multitude of graphics used in science exist and they may be grouped into one (or several) of the following categories.

- Conceptual illustrations are here considered "freehand" drawings to portray certain concepts qualitatively (see e.g., s-ink.org/ocean-plate-tectonics). Instead of directly representing data, they are visualising current knowledge or core concepts. It is therefore crucial that conceptual graphics are as accurate as possible. While sketches must be simple and details streamlined to a certain degree, they cannot be oversimplified and thereby misrepresent actual knowledge and lead to common misconceptions (for example, like a red, hot, and, therefore, molten Earth's mantle; the Earth's mantle is in fact a solid that deforms on geological timescales) that are difficult to counteract later on. Sketches are a particular type of conceptual illustration and, in particular, should be regularly revised and, if necessary, updated or removed.
- Data visualisations are data-based maps, charts, or graphs that quantify and relate the underlying data via graphical rulers, like an x-axis or a colour bar (see e.g., s-ink.org/oceanplate-age). For scientific data visualisations, data needs to be queen: the data must be represented without visual distortion, so that the readers can access (and interpret it) themselves, and the data should, in most cases, be a visually dominant aspect of a figure. If a data field is represented via colour and a colour bar, then the used colour map has to be perceptually uniform, meaning that the colour gradient between equally-spaced colour bar axis ticks must be equal (or change equally) to the eye all along the colour bar, as must the spacing between axis ticks be equal (or change equally) on a x-axis of a graph (Crameri, Shephard, and Heron, 2020).

- Animations are here considered motion graphics that can either be data-based (quantitative; see s-ink.org/mobile-lidmantle-convection), or not (qualitative). If animations are quantitative, they must fulfil the same quality standards as Data visualisations. If they are qualitative, they should be handled like Sketches.

- Artistic impressions are here artworks that represent a fictitious view on, and provide a feeling of, scientific concepts or different worlds (see e.g., s-ink.org/dynamic-planet-earth). The decreased emphasis of scientific accuracy differentiates Artistic impressions from Conceptual Illustrations. For example, a drawing of a liquid water-covered Martian landscape would be considered an Artistic impression.

- Icons are small, simple yet useful graphics that portray things beyond language borders. Several existing resources are available, one of them being the TheNounProject.com.

- Logos are graphical identifiers for private persons, groups, institutions, and initiatives (see e.g., s-ink.org/s-ink-logo). In contrast to other graphics, logos must only fulfil certain technical aspects like high resolution and are therefore open to unconditional artistic freedom.

- Posters are here display graphics (for example, for print) for scientific and/or public outreach, or simply science-related decoration (see e.g., s-ink.org/scientific-colour-map-poster). As posters can contain multiple panels constituting almost any type of content, they should undergo the same quality demands as outlined in the respective paragraphs. The poster itself, can, however, be freely designed, and should only consider effective visual knowledge transfer (e.g., by considering the betterposter approach; Morrison, 2019), if applicable.

- Graphical templates are the basic structures to a finished product enabling their time effective and graphically-guided creation. An example could be a presentation display (see e.g., s-ink.org/betterposter-poster-template) or x-y data template. As long as the graphical templates allow to fulfil all scienceproof standards mentioned in other paragraphs, they are open to unconditional artistic freedom. 


\section{'Mars topography'}

a. Data misrepresentation

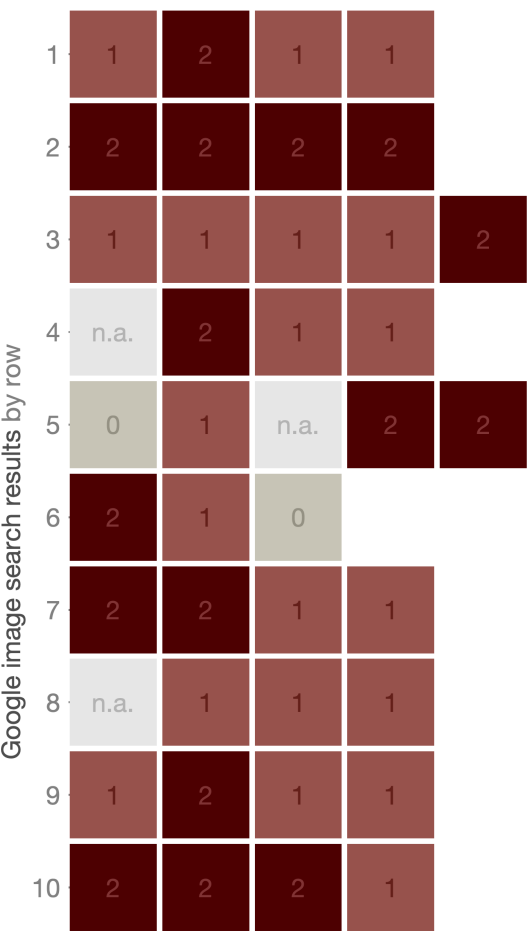

+1 : Perceptually distorted scale

+1 : Missing scale

\section{'Seismic hazard map'}

b. Visual inaccessibility

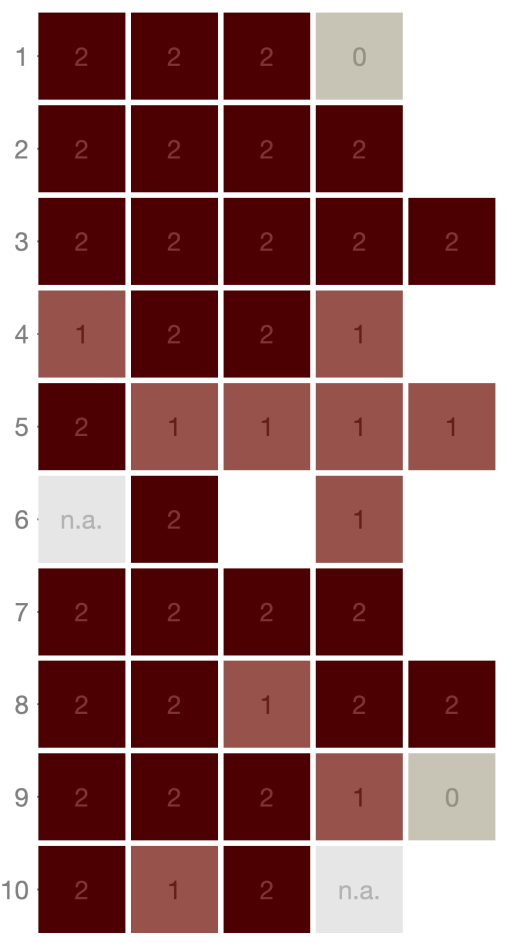

+1 : Colour-vision deficiency unfriendly

+1 : Unintuitive colour order
'Mantle convection'

C. Conceptual misrepresentation

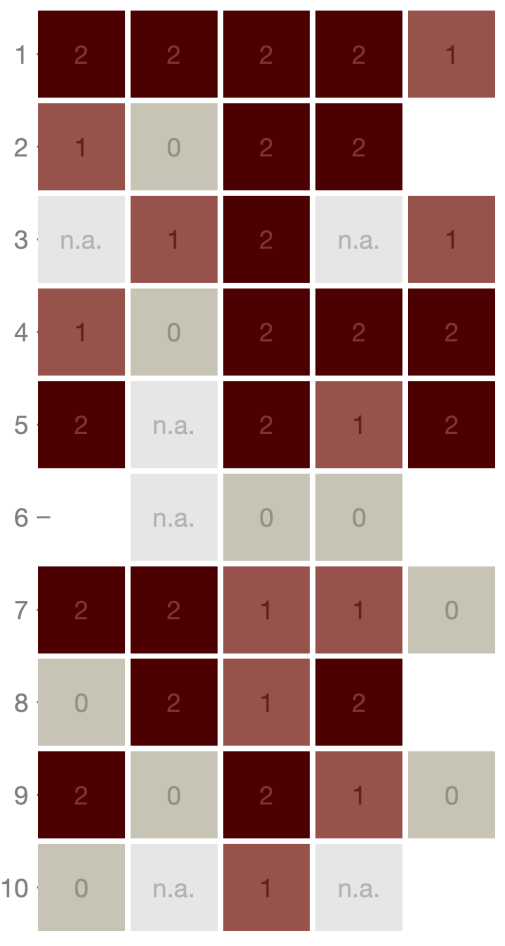

+1 : Molten mantle impression

+1 : Mantle plume-spreading ridge connection

Not applicable Acceptable Unacceptable

Figure I: Abundant flawed images in internet searches. Critical image pitfalls (in reddish colours) present in openly accessible images on key geoscience topics. Shown are counts for (a) perceptually distorted or even missing colour scales for the search 'Mars topography', (b) colourvision deficiency unreadable colour maps or unintuitive colour orders orders for the search 'Seismic hazard map', and (c) faulty, misleading concepts for a molten mantle or the direct connection of rising mantle plumes and spreading ridges for the search 'Mantle convection'. Individual counting panels are shown in order of appearance on search-results page with off-topic image results being marked not applicable (light grey). Results are based on an image search in December 202I as shown in Figure A.I. 
- Instructions are here guidelines for graphic designers to produce science-quality graphical products. A flow chart indicating what colour map type to use for a given data set would be such an example (see s-ink.org/colour-map-guideline).

- Graphical tools are code, algorithms, software, software libraries, and graphical parts thereof to create a finished product (see e.g., s-ink.org/delight-colour-inversion). The graphical tools in the collection must also (allow to) fulfil the quality standards outlined in the other paragraphs depending on their purpose. Graphical tools may require additional and detailed README-like documentation to ensure user ease.

- Photographs are here real-world images taken of scientifically relevant content. Great existing repositories like EGU imaggeo exist for photos and videos. Photographs should fulfil relevant graphical aspects as, for example, be clear in terms of the spatial scales portrayed.

- Videos are here real-world motion pictures taken of scientifically relevant content. As with photos, videos can be uploaded to and shared via repositories like EGU imaggeo. Videos should fulfil the same quality standards as the Photographs.

\section{Graphical quality measures}

Clarifying its category (see Section 3 ) is a first step to ensure highquality science graphics: it might, for example, not always be clear to a reader whether a figure shows actual data or not. Apart from grouping into categories, tagging individual graphics with several keywords further helps them to be found online. Optimally, science graphics would be offered in multiple versions that provide layered or alternative content for different use, such as labelling or language (see e.g., Section 4.2), or are visually adjusted for differing projection or on variable background (see e.g., Section 4.3). Where applicable, the language of content should be a common one, but additional language versions or translations would allow for an even broader use.

Vector graphics (i.e., those that can contain editable image portions, like .svg, .pdf, .ps, .eps) are generally most suitable for still graphics due to their higher visual quality and editability. However, raster formats (i.e., pixel images without editable image portions, like .jpeg, .png, .tiff, .gif) have also their advantages, like easy portability between various computer systems and software. In general, distributed graphics should be able to be opened by common and free image viewing software, and not require specific or paid software downloads (an exception would be the Graphical tools category).

The licensing for re-use should be clearly stated and open-access. Moreover, explanatory captions and other meta data (such as about creators and licences) should be provided alongside suggestions for citation and related references. Because both scientific knowledge and graphic-related technology is constantly evolving, graphics should be regularly updated, with the updates being communicated, or else, if outdated, be deleted.

While a particular graphic product might not convey information in the clearest way, high artistic quality tends to motivate the reader to spend more time looking at it (see e.g., Figure 3a). Whilst beauty is to some degree subjective, and in itself is hard to grasp scientifically, artists are well aware of the advantages of some of the nature-based mathematical patterns, like the golden ratio based on the Fibonacci sequence that produces eye-pleasing proportions. Similarly, various graphic design principles exist to make graphics more pleasing to look at. The colour theory, for example, offers a guideline for using multiple colours by defining primary, secondary, complementary, analogous, triadic, and other colour combinations. In today's flood of science output, unattractive graphics might simply not be looked at and are thereby rendered ineffective.

Artistically sound design is advantageous to attract readers, but graphics should also be readable to the general audience. Graphically, information is conveyed via our visual apparatus (i.e., the eye lens, the optical receptors named cone cells, the optical nerve, and the optical cortex), which is not uniform across all viewers (see e.g., Crameri, Shephard, and Heron, 2020). There is important variations in the perception of colours amongst human beings, with some colour-vision deficiencies (CVDs) disabling, for example, the differentiation of red and green at similar luminosity. While total colour blindness is fortunately rare, printing in black and white is still common amongst analogue paper readers. Graphics that are CVD-friendly and readable in grey-scale offer therefore a huge gain in visual accessibility (e.g., Figure 3 c and Figures A.2 and A.3).

Images appearing online profit from a short ALT-text (short for 'alternative text'), which is a concise description of the image in the event it cannot be viewed, and is important for accessibility. Further graphic accessibility comes from a clear visual layout, following design principles such as hierarchy (guiding the reader visually), balance (evenly laying out elements across the graphic), and contrast (differentiating individual elements). A good credo to follow is that when in doubt, simplicity should be chosen over complexity: Everything one can leave away in a visual (including text) should be taken away until only the essentials remain (e.g., Murchie and Diomede, 2020). The choice of font and typeface should also be based on both maximum clarity and aesthetics keeping in mind that individual fonts also transmit certain tones; common suggestions of (clear and serious) fonts in scientific graphic design include Helvetica, Futura, or Arial, but less common, but suitable options, like Open Sans, exist too.

In addition to a universally accessible design, scientific visualisation must ensure representing concepts and data in a clear and data-fair manner, preventing ambiguity and bias. While this seems obvious, the science community (editors and science communicators included) ran head-front into a literal visualisation crisis during the last couple of years: Data visualisation was predomi- 
nantly created with faulty colour axes leading to data misrepresentation and conceptual bias (see e.g., Figure A.4). Colour axes (a.k.a. colour bars) with uneven colour gradients (a.k.a. colour maps) introduce visual distortion and error to the underlying data of more than $7 \%$ of the displayed data range (Crameri, 2018a). Atypical for the science community, the warning call by a myriad of continuous scientific publications and visualisation experts (e.g., Robinson, 1960; Bertin, 1983; Ware, 1988; Tufte, 1990; Travis, 1991; Brewer, 1994; MacEachren, 1994; Rogowitz and Treinish, 1996; Brewer, 1997; Rogowitz and Treinish, 1998; Dent, Torguson, and Hodler, 1999; MacEachren, 2004; Light and Bartlein, 2004; HaghShenas et al., 2007; Borland and Ii, 2007; Bertin, 20II; Kovesi, 20I5; Hawkins, 2015; Thyng et al., 2016; Crameri, 2017; Monmonier, 2018; Crameri, Shephard, and Heron, 2020) has been widely ignored by non-experts, or even confronted with scientifically unfounded arguments, until recently.

A high standard for data representation is achieved, for example, by implementing the use of scientifically-derived colour palettes following the suggestions given in Crameri, Shephard, and Heron (2020) and the references therein. While representation (and not only interpretation) is an absolute necessity in scientific data visualisation, the use of outdated scientific concepts in graphical illustrations is crucial to avoid. Representing accurate concepts (as is done in Figure A.5) helps to eradicate common misconceptions such as the ones about a molten mantle or the direct dynamic connection between whole-mantle upwelling and spreading ridges at the surface (e.g., Crameri et al., 20I8; Crameri, Shephard, and Conrad, 2019).

High quality graphics are a crucial tool to narrow the currently widening gap between science and society (Makri, 2017). Below, we therefore outline powerful capacities of scientific graphic design represented by various, widely applicable examples.

\subsection{Specimen 1: Ensuring accessibility}

Firstly, we provide an example based on publicly-available data and re-plotted as an original figure. For this scenario, we use the age of the Earth's oceanic seafloor (ocean-plate age; Müller et al., 1997). To-date, this dataset has rarely been represented fairly and inclusively. To the contrary, by being commonly displayed with a rainbow-like colour chart, it contains visual artefacts and is unfit for colour-vision deficiencies. The prevalent use of rainbow for this dataset, and others, becomes clear through an online image search (similar to Figure A.I) or by using current versions of widelydistributed geoscience software.

Figure $2 \mathrm{a}$ is an openly available data visualisation that provides the ocean-plate age free of unfair and inaccessible colour combinations. The colour coded ocean-plate age data is readable for people with colour vision deficiency (Figure $2 \mathrm{~b}$ ) and even in black-andwhite prints (Figure 2c) thanks to the Scientific colour map batlow (Crameri, 2or8b). Moreover, the data is made the dominant aspect of the figure through the use of an interrupted Mollweide map projection (after Crameri et al., 2020) to effectively represent the world oceans.

\subsection{Specimen 2: Broadening applicability}

Secondly, we provide an example of an original, visually attracting figure. Time is synonymous with geology and there exists a multitude of artistic versions of the geologic time scale. While the table-like representation of geologic times and occurrences is certainly useful and clear to an interested viewer, it tends to appear rather unpleasing to a non-enthusiast's eye. Figure 3 is an original, artistic version of the geologic time scale that may not be the most accessible version (due to tilted text and non-linear time axis) compared to more standard rectangular versions (compare to Figures A.6 and A.7), but, thanks to its artistic flavour, it tends to also draw the non-enthusiast in for closer visual inspection of its content (see Figure 3a).

Importantly, the figure also illustrates how information-based graphics can provide flexibility to use (Figure $3 \mathrm{~b}-\mathrm{d}$ ) and accessibility to read (Figure $3 \mathrm{e}-\mathrm{g}$ ). This original graphic is provided as a so-called bare-bone layer too, providing only the key visual detail (here the time scale) to enable the users to alter and adjust the content to their specific intention. In some cases, the time axis might be more insightful when numbered as Million years ago from the present (Figure $3 \mathrm{~b}$ ). In other cases, labelling time as Million years since the start of something might be more suitable (Figure $3 \mathrm{c}$ ). The scientific content displayed onto the bare-bone layer may be varied too (i.e., replaced to not overload the graphics; Figure $3 \mathrm{~d}$ and e). And finally, other aspects like language or font-size may be changed, again to extend the graphic's usage.

\subsection{Specimen 3: Adding variability}

Closely related to the previous example, by broadening graphic applicability, the following graphic varies an image's appearance. Avoiding to bias our scientific understanding by a certain constantly repeated - and hence deeply ingrained - perspective on certain content should be avoided in science to reduce unnecessary mental barriers. World maps are a good and often discussed example as they introduced widespread misconception about the relative size or geometry of landmasses.

A multitude of map projections exist (Figure 4). Map projections can be divided into different families, which are cylindrical (e.g., Mercator; Figure 4c), conic (no example shown here), or azimuthal (e.g., Vertical Perspective Azimuthal; Figure 4b). Furthermore, map projections can be divided based on the properties they preserve, such as shape, distance, direction, scale, and area, and also based to the distortion to be minimised. Map projections are called equal-area (or previously homolographic), when they portray areas across it proportionally to the natural counterparts they represent (e.g., Mollweide; Figure 4a). Projections are called 


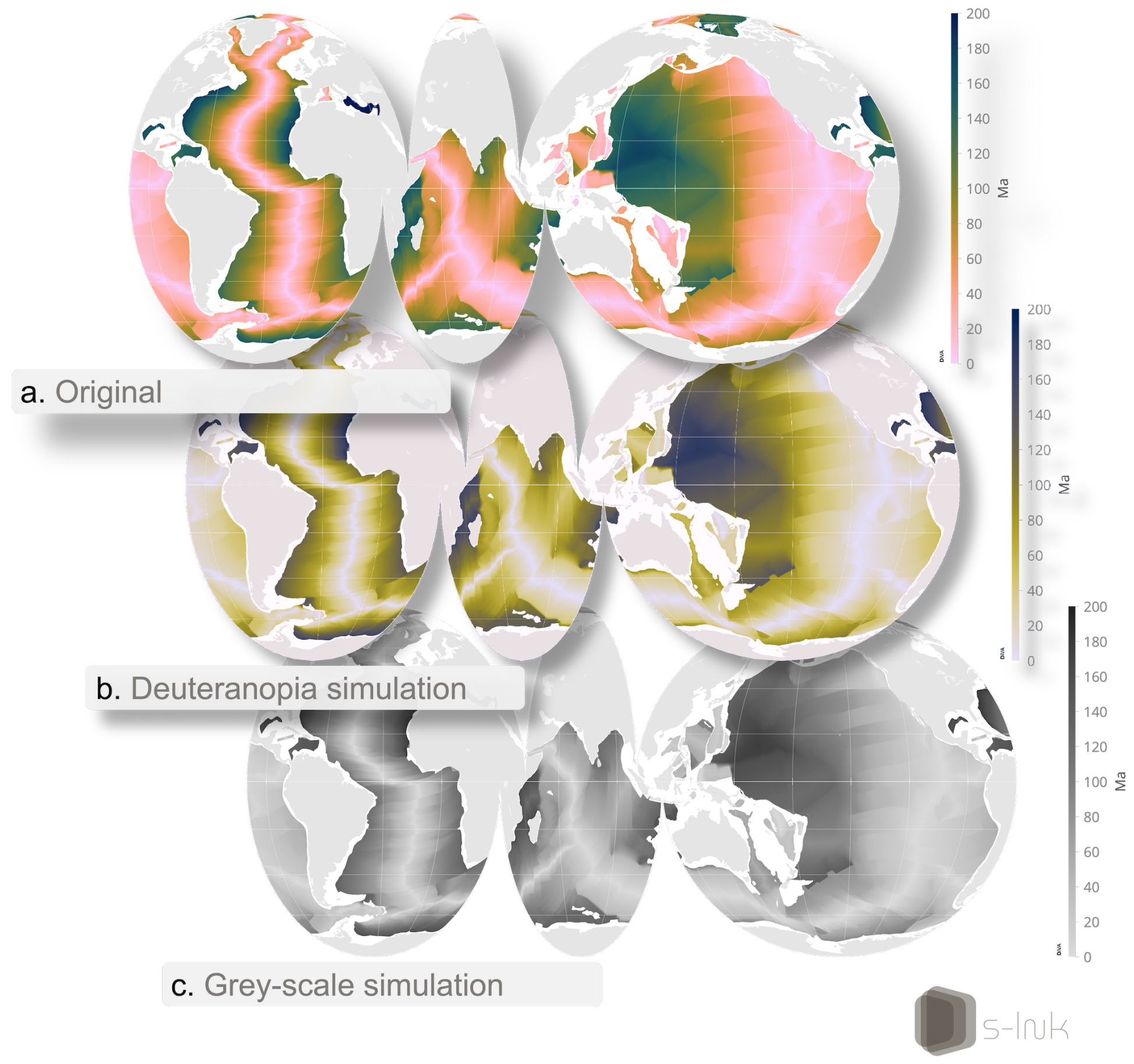

Figure 2: Accessible data visualisation. Data-true ocean-plate age visualisation, using the perceptually uniform batlow colour map from Crameri (20I8b), on an interrupted Mollweide map projection of the world oceans after Crameri et al. (2020), provided for effective information transfer (a) in its original form, as well as (b) for colour-vision deficient readers and (c) in grey-scale prints. Data from Müller et al. (1997). Specimen available at s-ink.org/ocean-plate-age. 


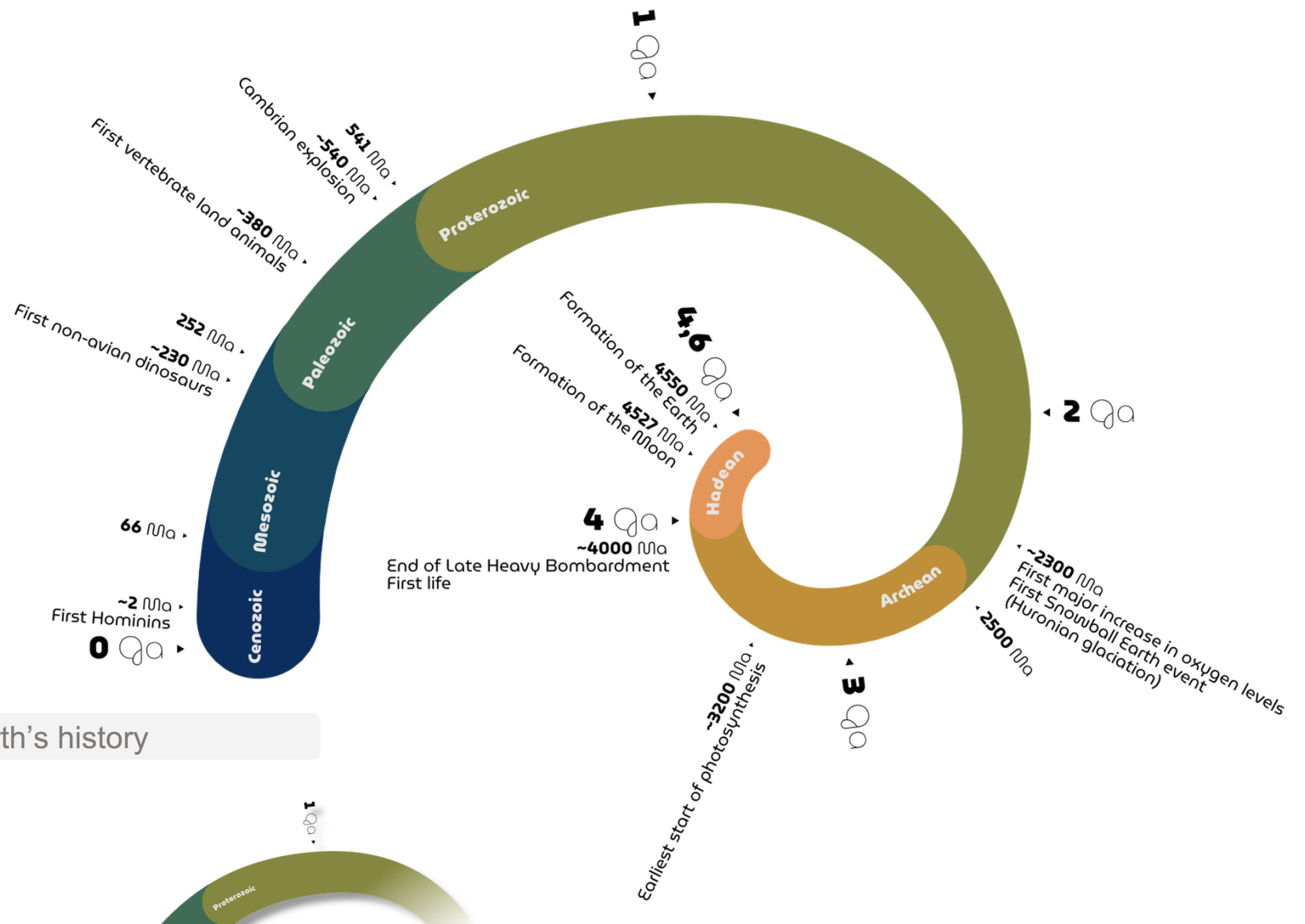

a. Earth's history

b. Barebone, time ago

c. Barebone, time since

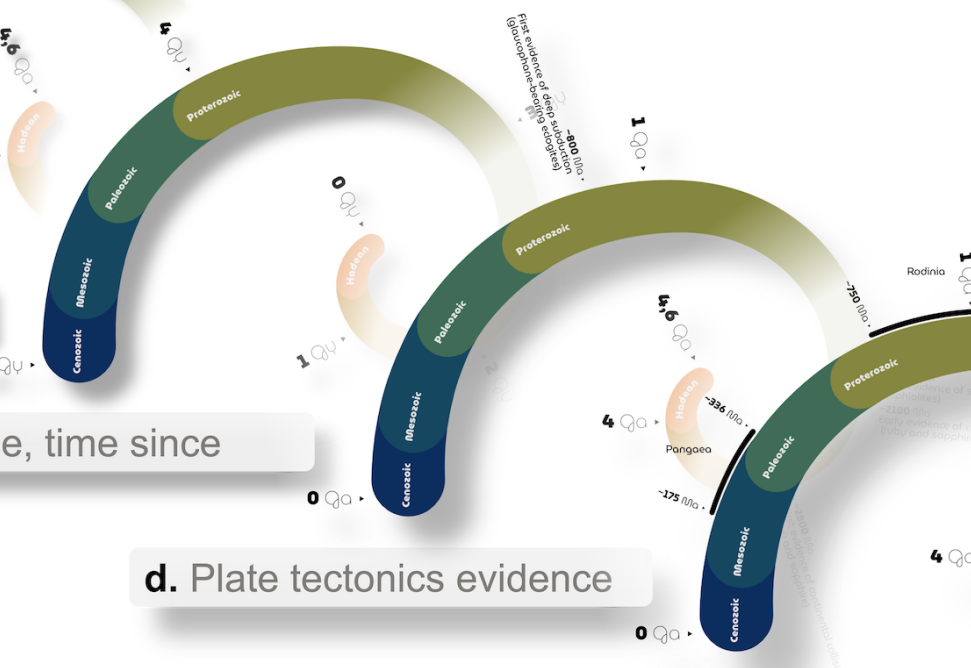

e. Earth's supercontinents

Figure 3: Layered-content graphics. (a) An artistic version of the geologic time scale that is suitable for a broad range of applications thanks to variable and adjustable layered content including (b) a template for later manual adjustment with the time axis formatted as time ago from present day and also (c) time since the beginning, and also with predefined alternative content like (d) prehistoric plate tectonic evidence or (e) reconstructed presence of supercontinents. Specimen available at s-ink.org/geologic-time-scale-artistic. 


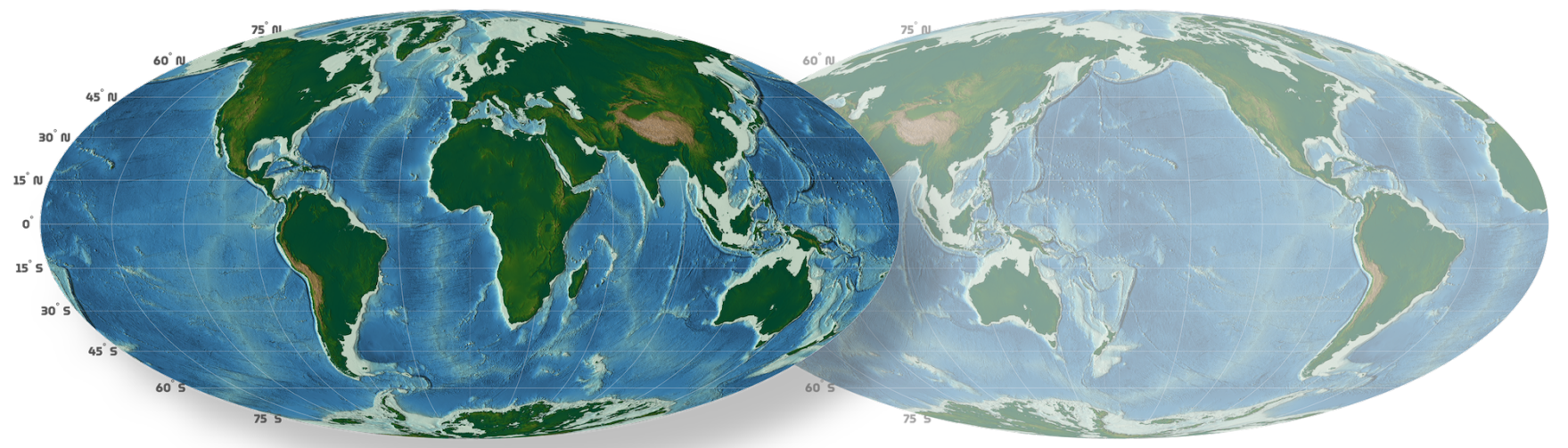

a. Mollweide

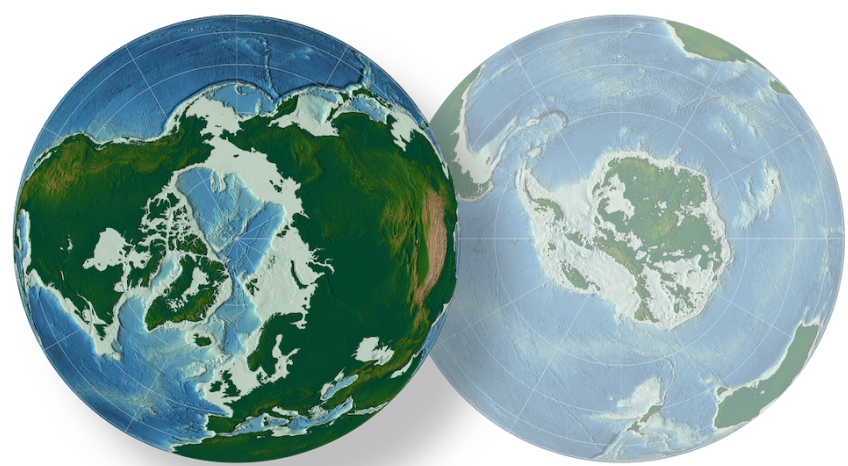

b. Vertical Perspective Azimuthal

d. One Ocean

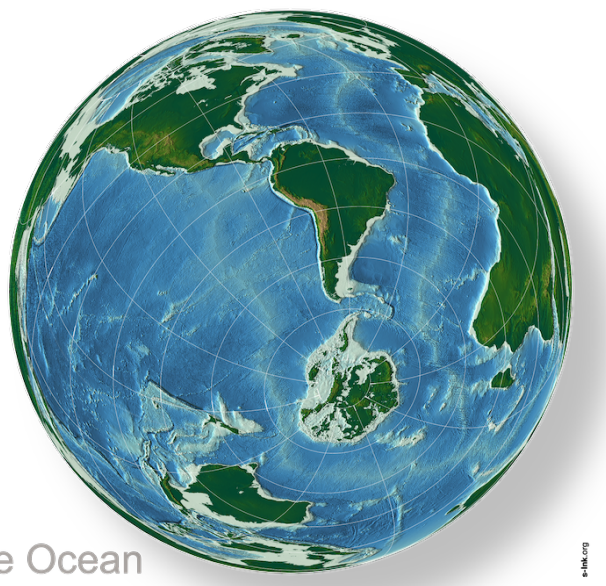

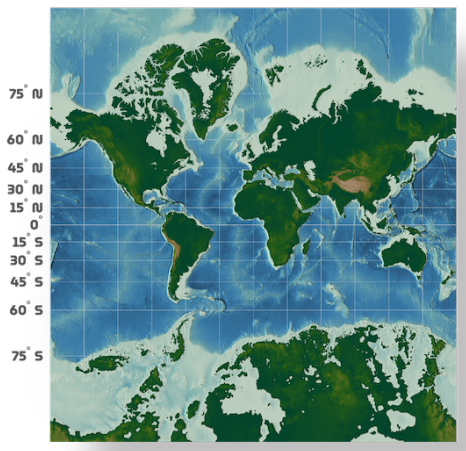

C. Mercator

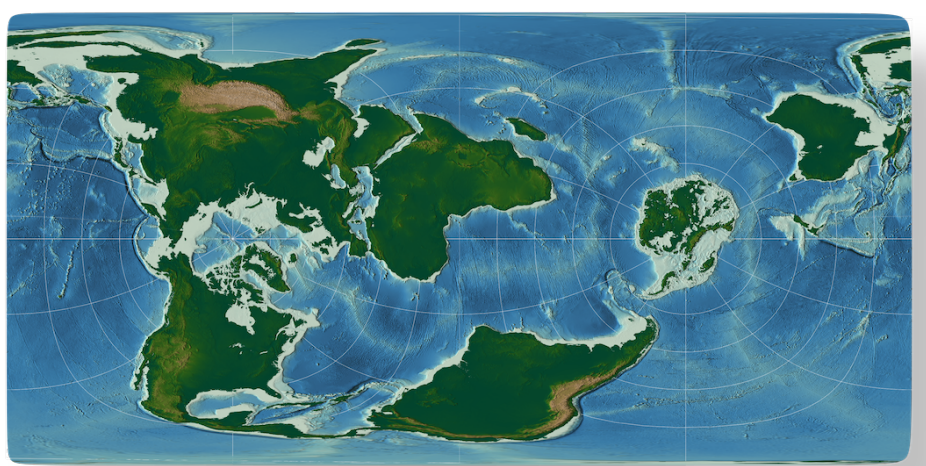

e. Cassini

Figure 4: Multiple presentation options for the same data set. Here shown is the surface elevation of the Earth on various map projections, which are (a) an equal-area Mollweide centred once at o and once at I8o degrees longitude, (b) an intuitive Vertical perspective azimuthal once centred on each pole, (c) a conformal Mercator, (d) a One Ocean, and (f) an equidistant Cassini. Specimen available at s-ink.org/surfacetopography-relief. 
conformal (previously orthomorphic) when the shape is preserved locally on a map (e.g., Mercator; Figure 4c). This is the case when the scale is the same in any direction and meridians (lines of longitude) and parallels (lines of latitude) cross themselves at right angles. No map can be both conformal and equal-area. A map projection is called equidistant, if distances from the centre (and from the centre only) of the projection to all other places on the map are preserved (e.g., Cassini; Figure 4e). In addition, maps are also described as equidistant when the separation between parallels is uniform. However, no map projection maintains distance proportionality in all directions from any arbitrary point.

Depending on the scientific task at hand, one map projection is likely more optimal than the other. In addition, for certain tasks, only some geographic areas are relevant, such as when only oceanic parts have to be shown (as in Figure 2). Custom maps like One Ocean (Figure 4d) might therefore be useful. Providing multiple map projection families and types of the same underlying data set therefore makes such graphics more versatile.

The other reason to provide multiple map versions is to prevent deeply ingrained perceptions of distorted geometries. For example, the relative size of Greenland with respect to Europe is widely misjudged, due to the widespread use of non-Equal-area map projections. Providing additional data set translations or orientations on a map does prevent perceptual and, possibly, conceptual bias risen from a certain appearance.

\subsection{Specimen 4: Facilitating creativity}

The next scenario is a graphic template to facilitate creating original graphics. Most academics attend large conferences to communicate their research. While digital and hybrid conference formats are becoming more common, the good old printed poster is still omnipresent. Large meetings with hundreds or thousands of attendants and presentations impede in-depth discussions. It is therefore as crucial to seek relevant scientific findings effectively, as it is to promote a scientific finding to as many interested peers as possible. Moreover, busy academic schedules often leave little room for carefully designing every single poster.

The 'betterposter' design (Morrison, 2019) offers a quick-tocreate design solution to effectively communicate research findings at large conferences with busy attendees. Powerpoint and Keynote templates (see Figure 5) enable (re-)creating a poster in the given design. The visually prominent focus area allows to directly access the main finding discussed on the given poster from a distance. Details are arranged on the side of the poster area to guide interested viewers coming up-close through more in-depth aspects like the methods.

\subsection{Specimen 5: Spreading capability}

The fifth example is a scenario of a figure-editing software program. Such a graphic tool is factually packaging graphic skills (if only in form of a tool) and makes them readily available to the wider science community.

Science communication includes sharing scientific work in different formats. Original publications are often reformatted into, for example, oral presentations, news articles, or twitter posts. Doing so, often demands to re-use certain graphics. However, most graphics are intended for, and work best with, the specific canvas they have been produced for. An effective way to adjust graphics for different purposes is therefore not only helpful to the communicator, but ultimately improves science communication itself.

Since the perception of foreground colours strongly depends on the background colour (see e.g., Crameri, Shephard, and Heron, 2020), image colour inversion is such a critical adjustment to be undertaken for displaying a certain graphic effectively on a variety of different canvases. For example, the image colour inversion tool deLight (Crameri, 2020) enables science communicators to adjust the colours of an existing image file from working effectively with a light background to work effectively with a dark background (Figure 6). Apart from some other functionality, the MatLab tool offers to either invert all colours based on their lightness or, else, to invert only the grey-scale image portions. While using MatLab itself necessitates a licence, the software code is open source (i.e., readable) and therefore reproducible, extendable, and free to use.

After creating science-grade graphics, the next, important step is to make them readily available. All above examples of science graphics, and many more, are now freely and readily available via a novel online collection.

\section{The s-Ink collection}

Here, we introduce s-Ink (From Source To Ink, s-Ink.org, a pun on the geological method of source-to-sink), an online collection to enable easy access to openly-licensed, visually engaging, and science-quality graphics that are of wide use. The collection offers a convenient and time-saving workflow in form of a user-friendly online platform (Figure 7) to find suitable graphics for conveying scientific aspects in, for example, presentations, proposals, publications, editorial pieces, and news and Wikipedia articles. Moreover, s-Ink supports artistically-inclined scientists that often tend to end up spending a significant amount of their work-time crafting graphics for their peers; their efforts are now visible and shared with the entire community under a clearly stated license, so that their individual contribution is acknowledged. The content is downloadable either directly from the s-Ink website or via a link to an external site or repository (for example, in the case of large or pre-existing content).

Apart from being grouped into categories as outlined in Section 3, products offered via s-Ink are tagged with several keywords to facilitate finding graphical content within the collection. Both keywords and categories are easily accessible and searchable from the webpage front, accompanying an actual text search tool (Figure 


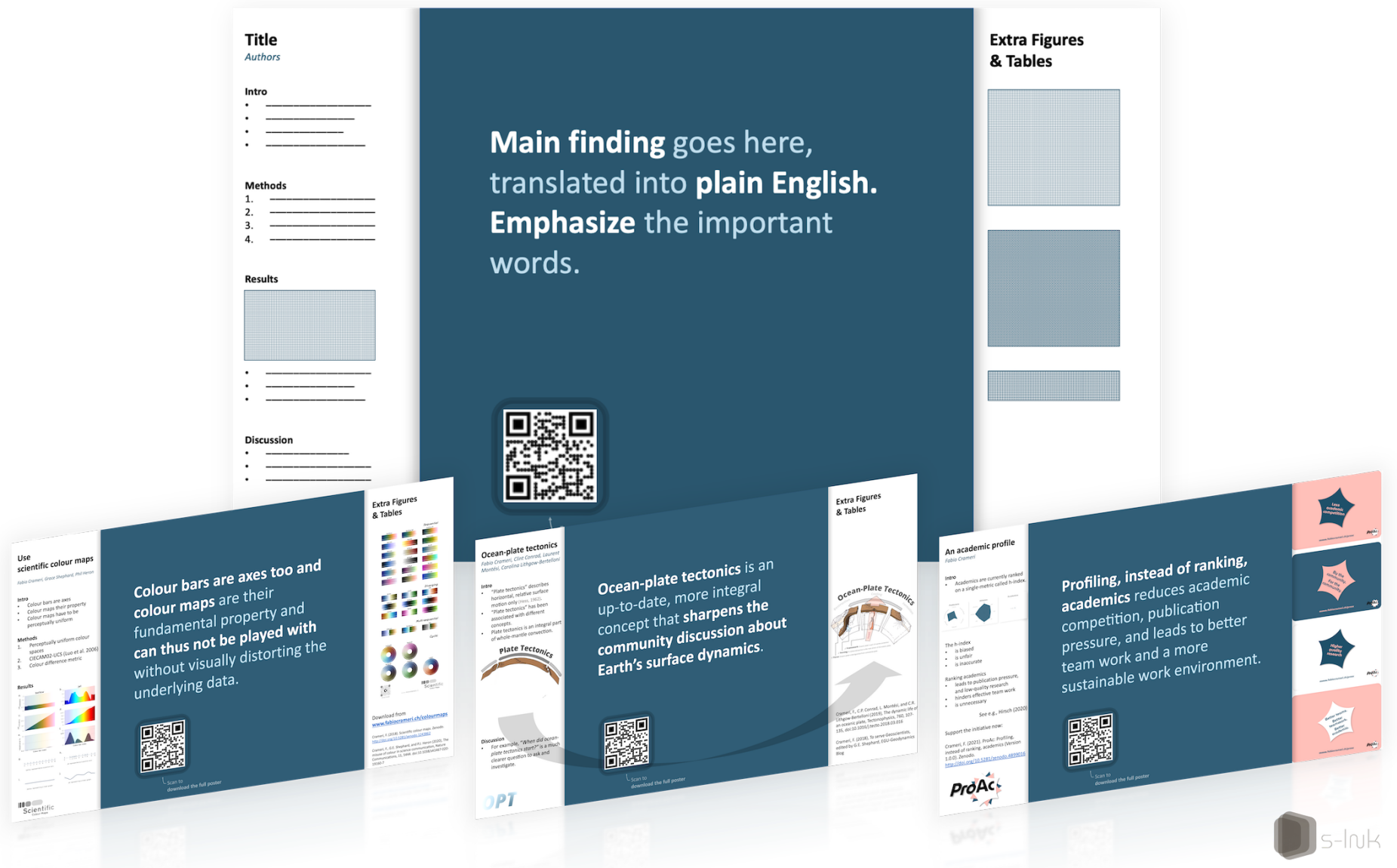

Figure 5: 'Betterposter' poster template. A poster design template that facilitates quick creation of poster designs that allow new scientific findings to be effectively communicated at large meetings. The template closely follows Mike Morrison's poster design suggestion (Morrison, 2019). Specimen available at s-ink.org/betterposter-poster-template. 


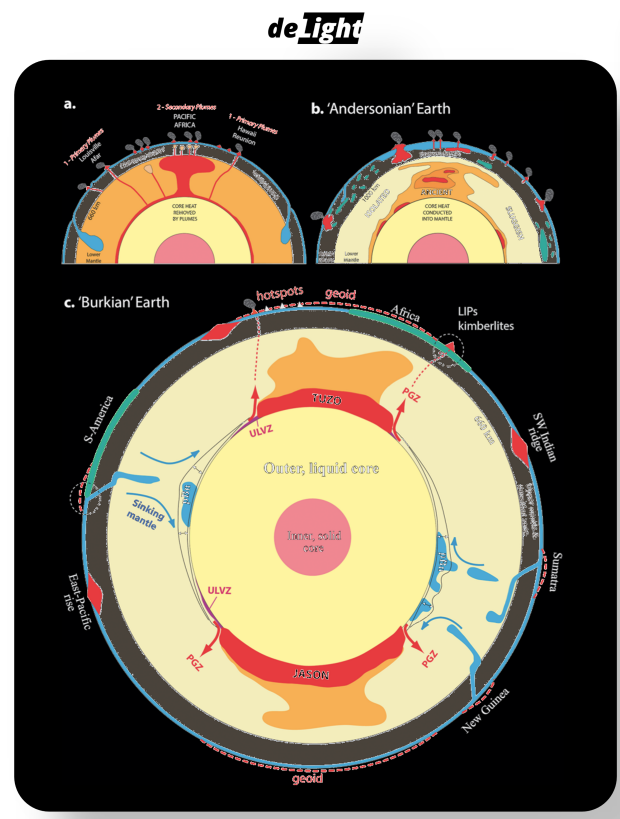

b. 'Maintain colours' mode
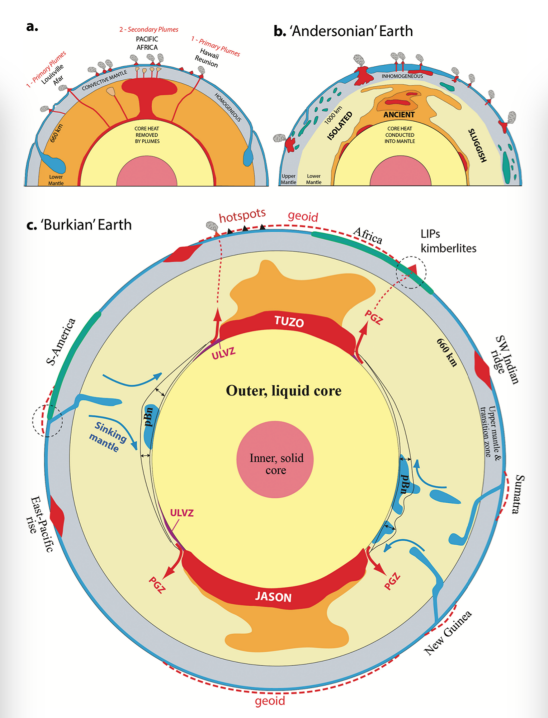

a. Original

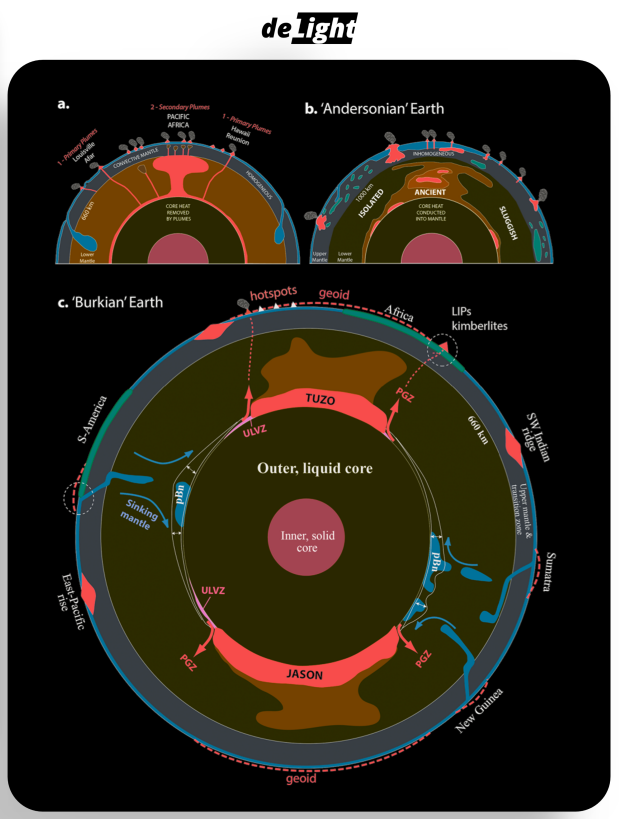

c. 'Invert all' mode

Figure 6: Colour image inversion tool. The MatLab software deLight (Crameri, 2020) empowers even a non-expert user to invert colour of (a) any original image, here an example figure from Torsvik et al. (2016), either by inverting (b) only the grey-scale portions or (c) all colours in a clever way to maintain intuitive colouring. This capability ensures effective visual information transfer also on different canvases like dark presentation screens. Specimen available at s-ink.org/delight-colour-inversion. 


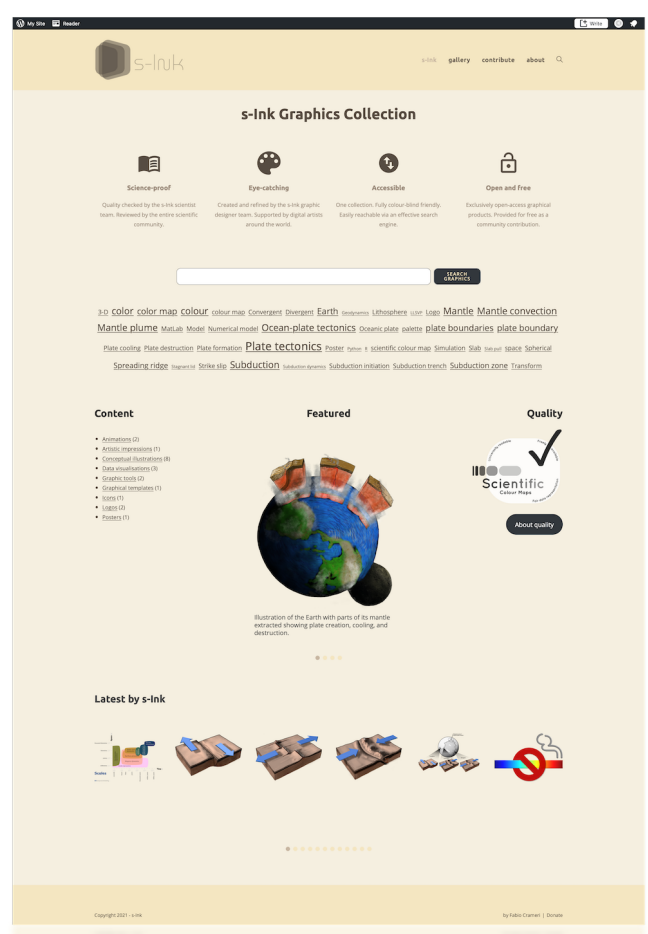

a. Front page

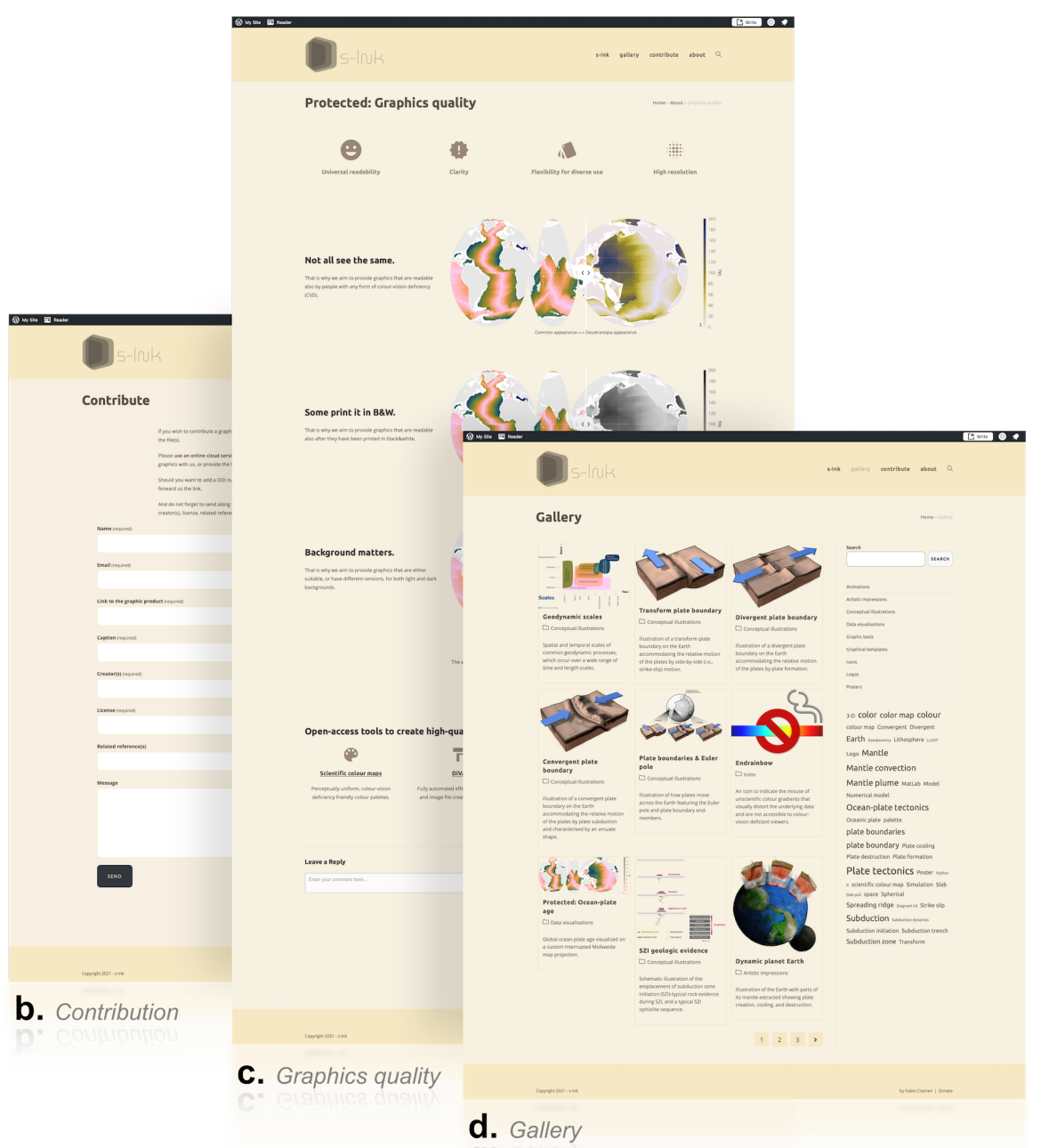

Figure 7: s-Ink.org. The carefully designed online platform includes (a) a clean front page, with direct access to the powerful search engine, (b) a submission form for contributions by the larger community, scientists and artists alike, that undergo scientific and artistic quality checks as outlined on a (c) graphics quality page. Suitable graphics then finally appear in (d) the online gallery for everyone to access. The visual design of the online platform is inspired by the Art Gallery of New South Wales and puts both the user experience and the graphical content into focus. 
7), as outlined in detail in Section 5.3.

If possible, s-Ink products are offered in multiple versions that provide layered or alternative content for different use such as labelling or language (see e.g., Section 4.2), or visually adjusted for differing projection or on variable background (see e.g., Section 4.3). Where applicable, the language of content should be English (UK or US), but additional language versions or translations are possible to be uploaded alongside the original version. Vector graphics (i.e., those that can contain editable image portions, like .svg, .pdf, .ps, .eps) are favoured for still graphics due to their higher visual quality and editability. However, raster formats (i.e., pixel images without editable image portions, like .jpeg, .png, .tiff, .gif) are also distributed via s-Ink. Similarly, there is little restriction to other commonly distributed file formats for other types of graphics. Content should be able to be opened by common and free image viewing software, and not require specific or paid software downloads (an exception would be the Graphical tools category).

The downloadable files are either stored in the s-Ink collection (i.e., hosted on the s-Ink servers) or, else, linked to other repositories (i.e., via a hyperlink to an external website). Products distributed via s-Ink are distributed under a specified open-access licence and hence available to everyone. Moreover, explanatory captions and other metadata about creators and licences are provided alongside suggestions for the citation and any related references. A guide (via an instructive submission form) and FAQ (s-ink.org/faq) are provided prior to submission, because the submitter must gather and confirm necessary copyright and/or licenses.

Because both scientific knowledge and graphic-related technology is constantly evolving, s-Ink products can be regularly updated, with the updates being communicated through versioning based on "last update" dates or version numbers. Outdated content that cannot be updated may be deleted from the collection in the future.

The s-Ink collection exclusively provides, data-true and accessible graphics, to the extent possible, so that users can be confident that knowledge and data is displayed to as many readers as possible and with minimum visual distortion.

In general, the s-Ink collection contains, and is open to, sciencerelated content of several graphical types as long as they are of wide interest, use, and accessibility. Specific quality standards will apply and submissions will be guided with a check-list. The current version of s-Ink contains several submissions related to the solid Earth sciences, but all geoscience-related topics (and even other science topics) are welcomed.

\subsection{Community contributions}

Everyone can provide or suggest open-access graphics for the online collection. Suggestions can be submitted via the online platform, sInk.org/contribute (Figure $7 \mathrm{c}$ ), by filling out the submission form provided. Before publication on s-Ink, suggested graphics will be initially reviewed regarding their usability to a wide audience and on the basis of s-Ink's artistic and scientific quality standards. The latter are conveniently provided in form of a submission check-list (e.g., on s-ink.org/contribute; see also Section 4). The moderators may request the submitter to make changes to the contribution before it appears in the s-Ink collection.

\subsection{Community feedback}

The s-Ink graphics being accessible for everyone opens a truly powerful avenue: feedback by the entire education, science, and art communities is enabled. After being exhibited in the s-Ink collection, any graphical content in the online collection is open to continuous community feedback, which acts as a reliable and unbiased quality control mechanism.

Feedback is exchanged via the comment option at the bottom of each individual graphic's webpage and necessitates a simple login, to prevent spamming. Following constructive comments, graphic products are supposed to be revised by the individual creators, if needed and possible (e.g., a data visualisation based on a numerical simulation is possibly more challenging to change content-wise than a sketch is). A user can therefore be confident of the good scientific and graphic quality and wide visual accessibility of the s-Ink content.

\subsection{Accessing collection content}

The s-Ink collection brings together widely useful, key (geo)scientific visual products and makes them readily and freely accessible via the centralised online web platform $s$-Ink.org. The easy access to content is one of its key pillars. It manifests itself by a clear and streamlined web design with an optimised search function, and by the absence of pay walls.

The s-Ink web platform aims to provide a self-explanatory user interface that offers a familiar environment already upon its first use, both on desktop computers and mobile devices. At the centre of the interface design is the top (or side) menu bar that includes links to all main pages and access to the search functionality. The search engine - the most important element for a personalised access to the s-Ink collection - is, the most visually prominent feature on the front page (Figure 7a). It is accompanied by a tag cloud, offering a topically assisted suggested search option, as well as a category list, to scan through a certain category of graphical products. Including the featured content, a 'latest by s-Ink' suggestion, and an entire gallery page (Figure $7 \mathrm{~b}$ ), an efficient access to specific content is thereby guaranteed.

The page design is fluid, and adjusts seamlessly, from a large desktop screen size to a mobile device screen size. Spanning all common screen sizes is an always-on 'page up' button giving easy access to the menu and the search functionality. The website's colour design is carefully crafted to be both visually appealing and effective in presenting (and highlighting) the individual graphical 
products, whether they may have a white, black, or transparent background.

A large collection is only useful if the content can be accessed efficiently and effortlessly. At the heart of s-Ink online platform is therefore a powerful search engine. It provides, for example, so-called 'fuzzy matching', that effectively matches partial words, if complete words do not match. Moreover, search results are sorted in the order of relevance, and not date. The search engine can be readily accessed via the s-Ink Front page (Figure $7 \mathrm{a}$ ), or the Gallery (Figure $7 \mathrm{~b}$ ). A page universal search panel is provided in the top menu for the convenience of the user.

\subsection{Using collection content}

One of the intentions of the s-Ink collection is to facilitate the use and creation of science-related visuals for academic purposes. These include for journal articles, books, proposals, teaching, presenting, outreach, and even creating new graphic tools. Therefore, all graphical products in the online collection will be openly accessible, but licensed individually to grant recognition of the time and work invested by their creators. Using s-Ink content will therefore be subject to specific licensing. The most common licenses used (e.g., CC 4.0 , creativecommons.org/licenses/by/4.o/, or MIT, opensource.org/licenses/MIT) necessitate to acknowledge the creator(s) in the same way it is common standard across all science disciplines for other academic products. To alter the graphic content of the individual products after download, it must be allowed by the given licence. Moreover, the s-Ink collection curation, storage, and free-of-charge access, should be acknowledged in addition (see Section 5.5).

\subsection{Collection curation and maintenance}

The s-Ink collection will update, improve, and extend its content over time. Everyone can suggest high-quality (see Section 5) graphics of wide interest to be added to the collection, and provide feedback on existing content. To minimise the storage needs on the ever-growing online collection itself, graphical content may also be linked from external repositories. Artists are also encouraged to put widely-used graphics on permanent repositories like Zenodo (zenodo.org), which additionally facilitates versioning and copyright attribution. The costs accompanying the online platform maintenance are currently covered by an institutional contribution, and filed under a community contribution. On the long term, however, the s-Ink collection will rely on sponsoring by academic institutions and/or donations (s-ink.org/donate).

\section{Closing}

Science-grade graphics are uncommon, but unmistakably an invaluable tool to scientists. By upholding key graphic design principles, the From-Source-To-Ink $(s-I n k)$ initiative provides an effective means to distribute (geo-)scientific knowledge visually amongst peers, but also to the general public. Thereby, it enhances visual science communication for both upcoming research and outreach. Importantly, providing the collection content under a clear creator's license is a crucial step forward towards granting the academic recognition scientific artists deserve. As an ultimate result, we believe, s-Ink will put the ink back to the source, the pen back into the hand of the (geo-)scientist, and the scientific knowledge back to the minds of the general public.
Author contributions. FC and GES conceived the project. FC designed both the manuscript figures and the online collection with feedback from GES and EOS. All authors contributed original graphics to the s-Ink collection, and contributed to the manuscript.

Competing interests. The Authors declare no competing interests.

Acknowledgements. While FC is mainly privately funded, all authors acknowledge support from the Research Council of Norway through its Centers of Excellence funding scheme, Project Number 223272. GES is funded by the Research Council of Norway's Young Research Talent project scheme (Project number 326238 - POLARIS, Evolution of the Arctic in deep time). Valentina Magni contributed ideas to the original project proposal. We are grateful to Carmen Gaina and The Centre for Earth Evolution and Dynamics (CEED) for the support for this early-career project via the YoungCEED programme. 


\section{'Mars topography'}

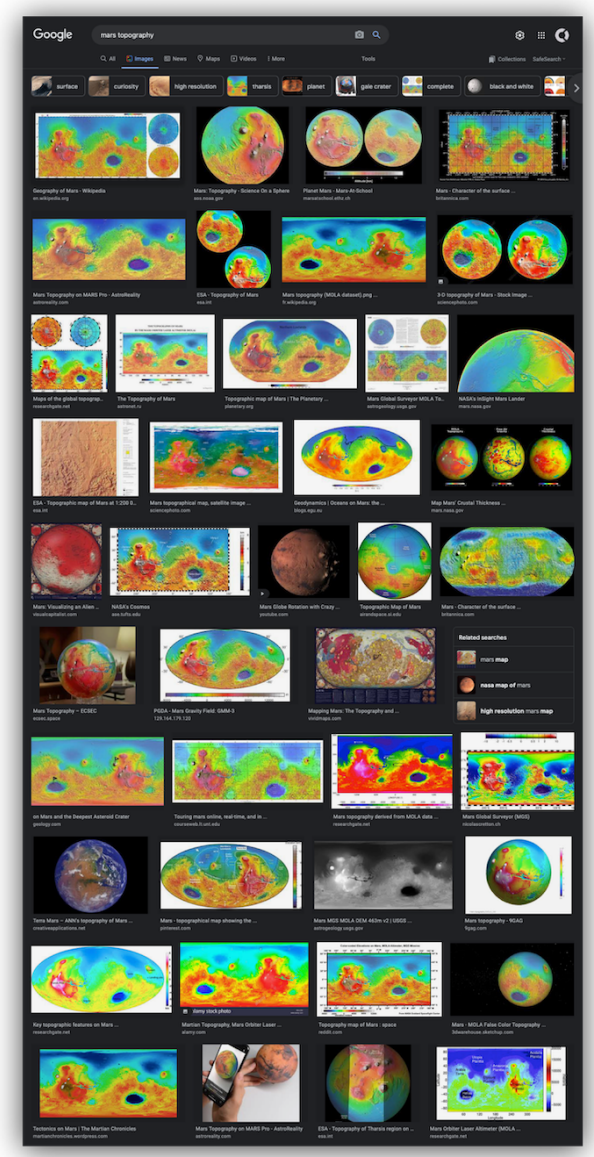

'Seismic hazard map'

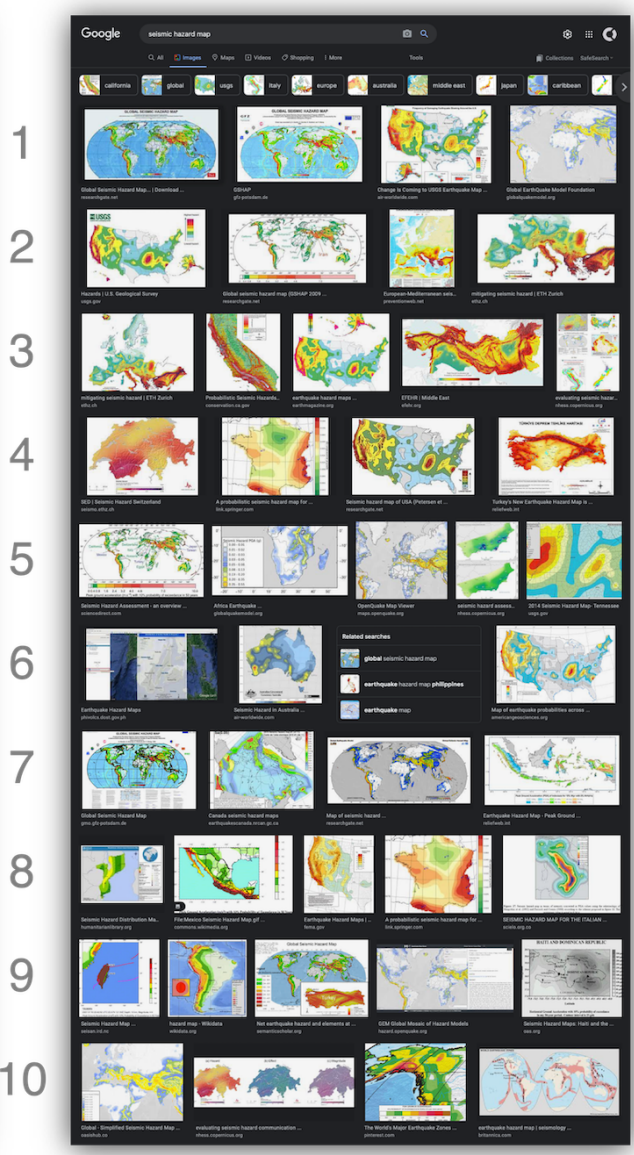

'Mantle convection'

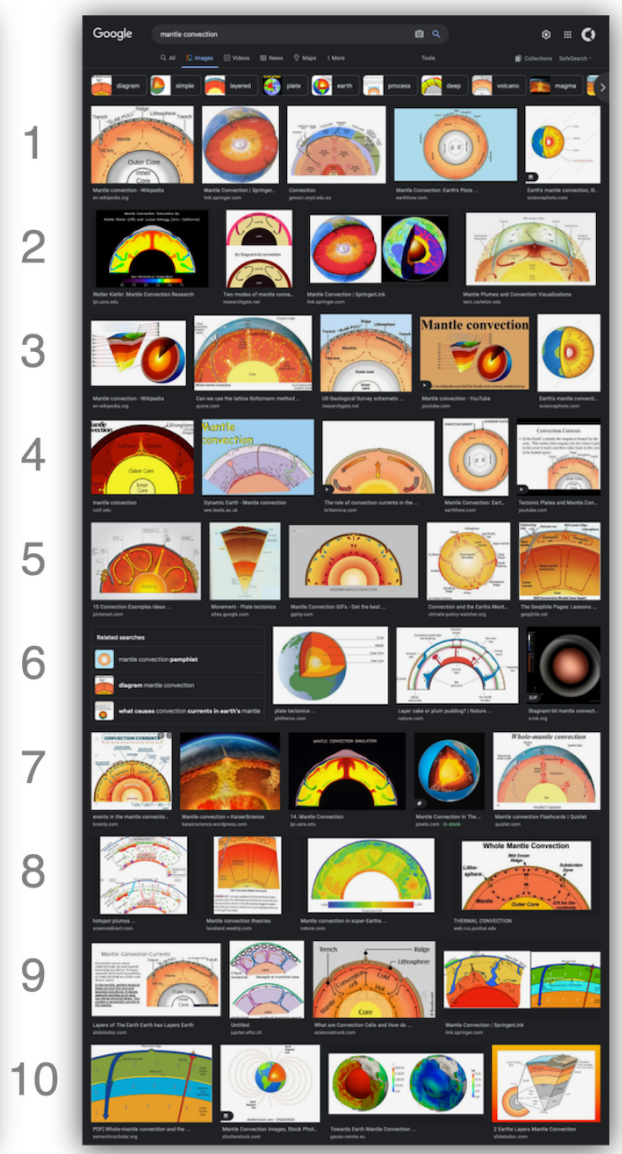

Figure A.I: Image searches for Earth Science key features. As of the year 202I, data-distorting and inaccessible colour maps and outdated, misleading scientific concepts in science communication are prevalent for image searches like 'Mars topography', 'Seismic hazard', and 'Mantle convection' (see analysis presented in Figure I). Effective high-quality alternatives are available via s-Ink at s-ink.org/mars-topography, s-ink.org/seismic-hazard, and s-ink.org/mantle-convection, respectively. 


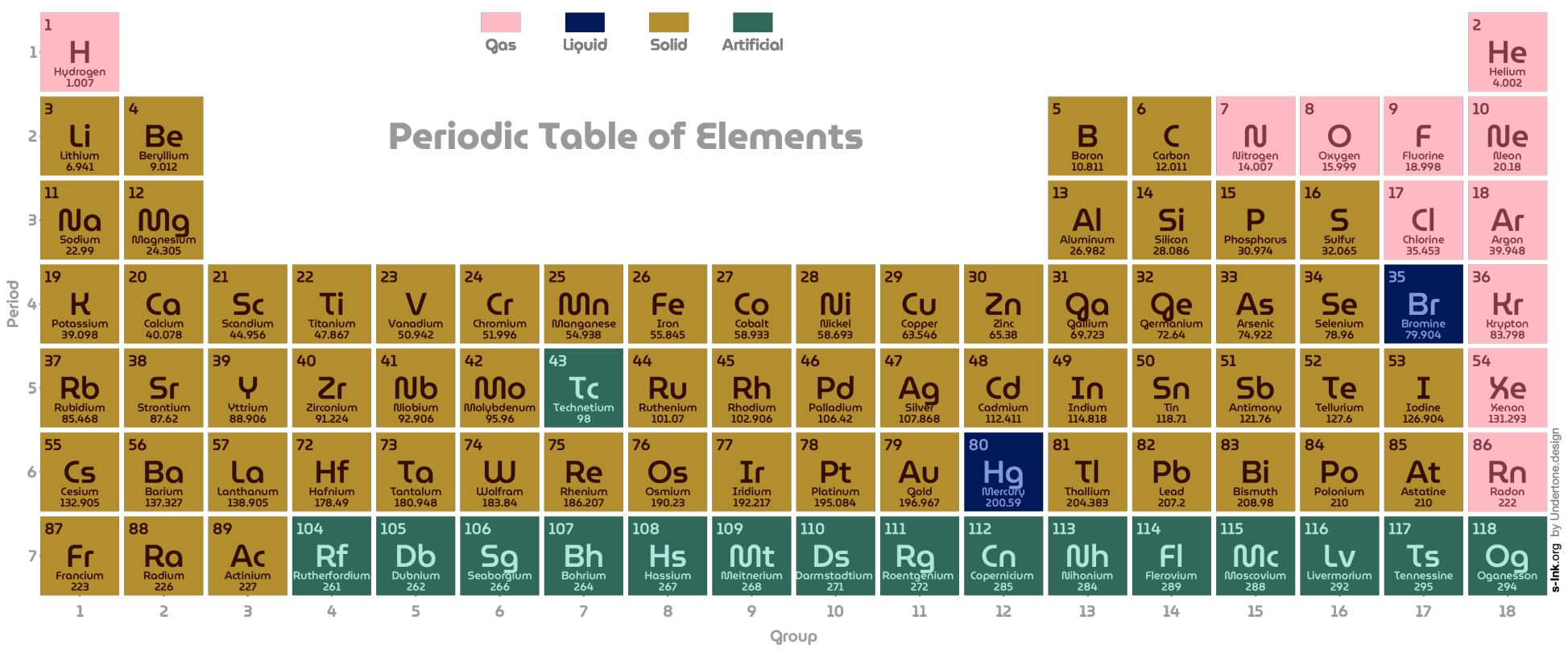

Figure A.2: Colour-vision deficiency friendly Periodic Table. Inclusive Periodic Table of Elements including atomic numbers, element symbols and names, atomic mass, and period and group. Coloured are the different phases of individual elements at room temperature. Coloured with the categorical scientific batlow colour map from Crameri (2or8b). Specimen available at s-ink.org/periodic-table-of-elements.

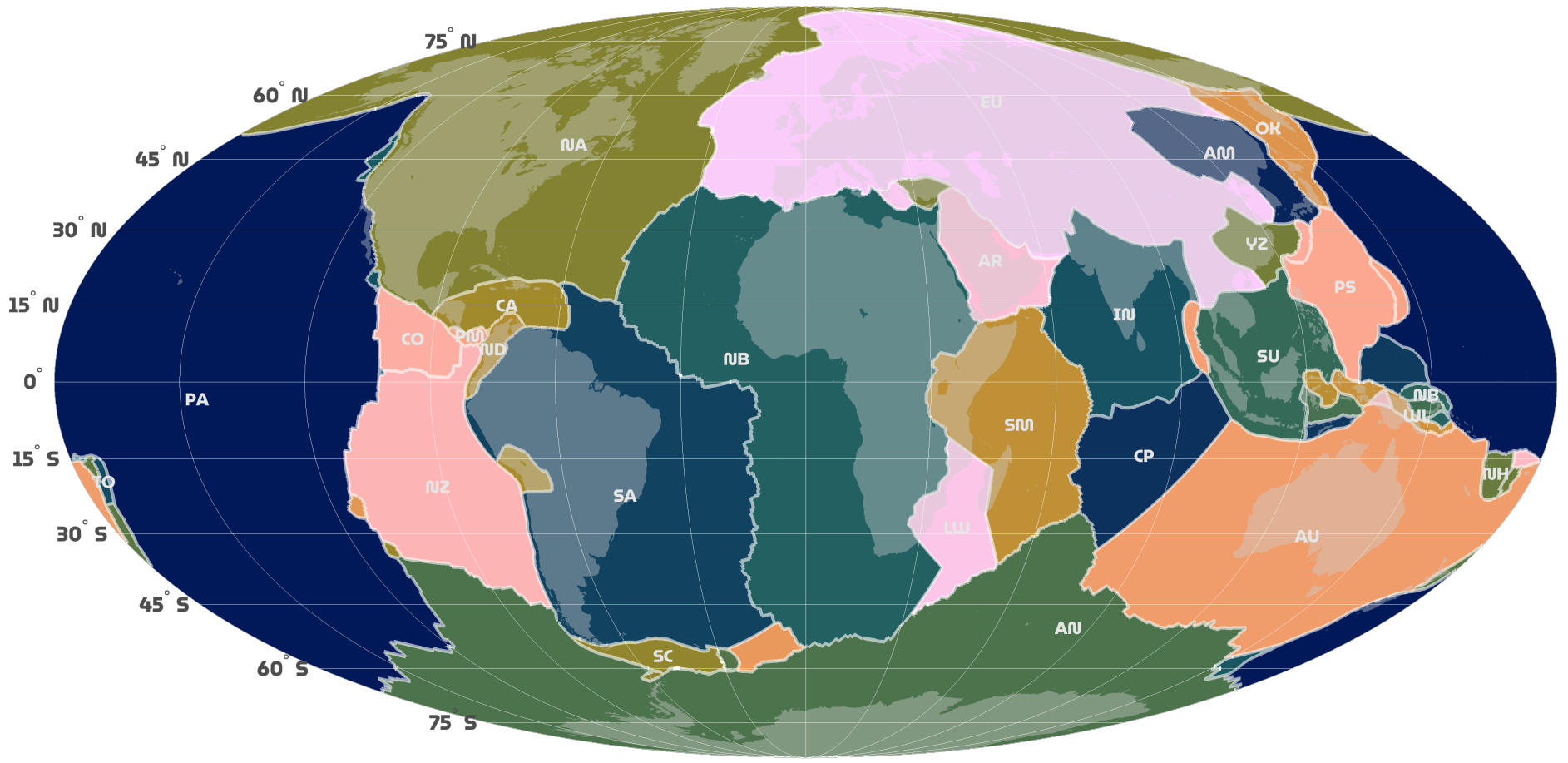

Figure A.3: Colour-vision deficiency friendly tectonic plates map. Inclusive map of the tectonic plates of the Earth, consisting of 56 individual plates named according to abbreviations given in Argus, Gordon, and DeMets (20II). Coloured with the categorical scientific batlow colour map from Crameri (20I8b). Specimen available at s-ink.org/tectonic-plates-simple. 


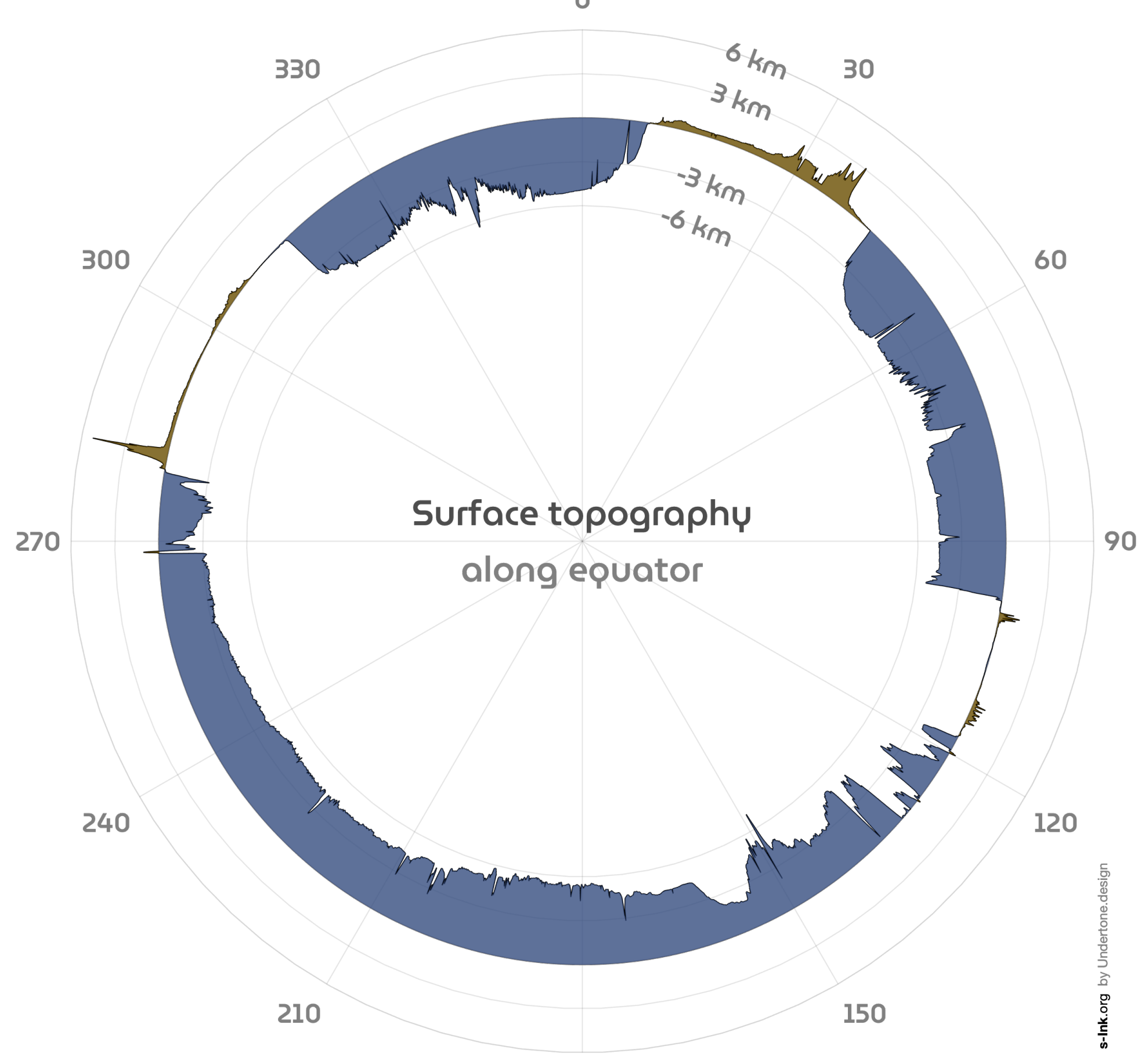

180

Figure A.4: Surface elevation along equator. Vertically exaggerated surface topography highlighting the height of mountains and the somewhat surprising depth of oceans thanks to fair data representation, in contrast to currently common surface topography colour maps that exaggerate low-elevation land topography compared to ocean bathymetry leading to widespread bias of the underlying dataset (Crameri, Shephard, and Heron, 2020). Specimen available at s-ink.org/surface-topography-cross-section. 


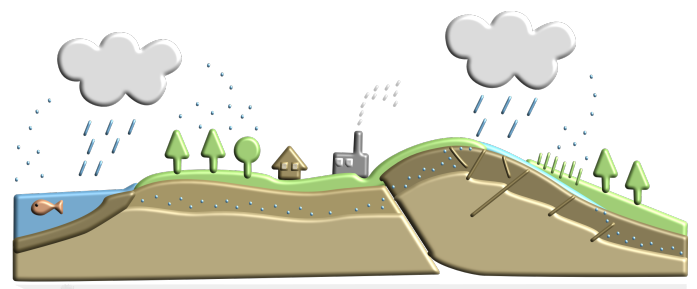

Figure A.5: Earth-system processes. A schematic highlighting some of the most relevant Earth processes occurring in and on the dynamic, presentday-style Earth, which generates and erases geologic records of its transforming states and is now experiencing unprecedented environmental change. An accurate, up-to-date depiction of features, like a solid (and not molten) mantle, and processes, like mantle convection with spreading ridges not being directly linked to upwelling mantle plumes, is critical to science outreach. Not all displayed individual features are to scale. Specimen available at s-ink.org/earth-processes. 


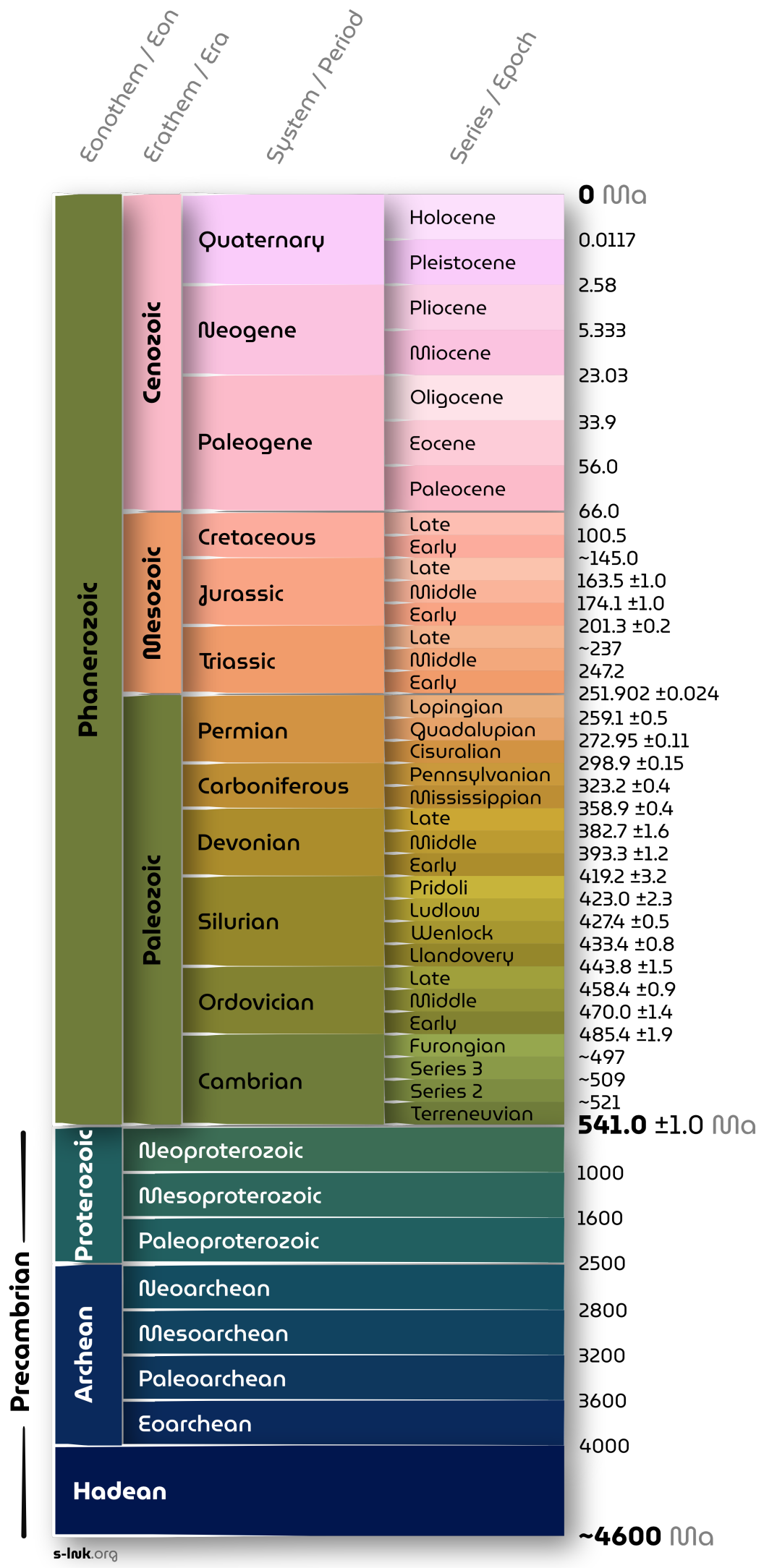

Figure A.6: Geologic time scale. As an alternative to Figure 3, the colour-vision deficiency friendly geologic time scale lists individual epochs, periods, eras, and eons accurately. Coloured with the categorical scientific batlow colour map from Crameri (2or8b). Specimen available at s-ink.org/geologic-time-scale. 


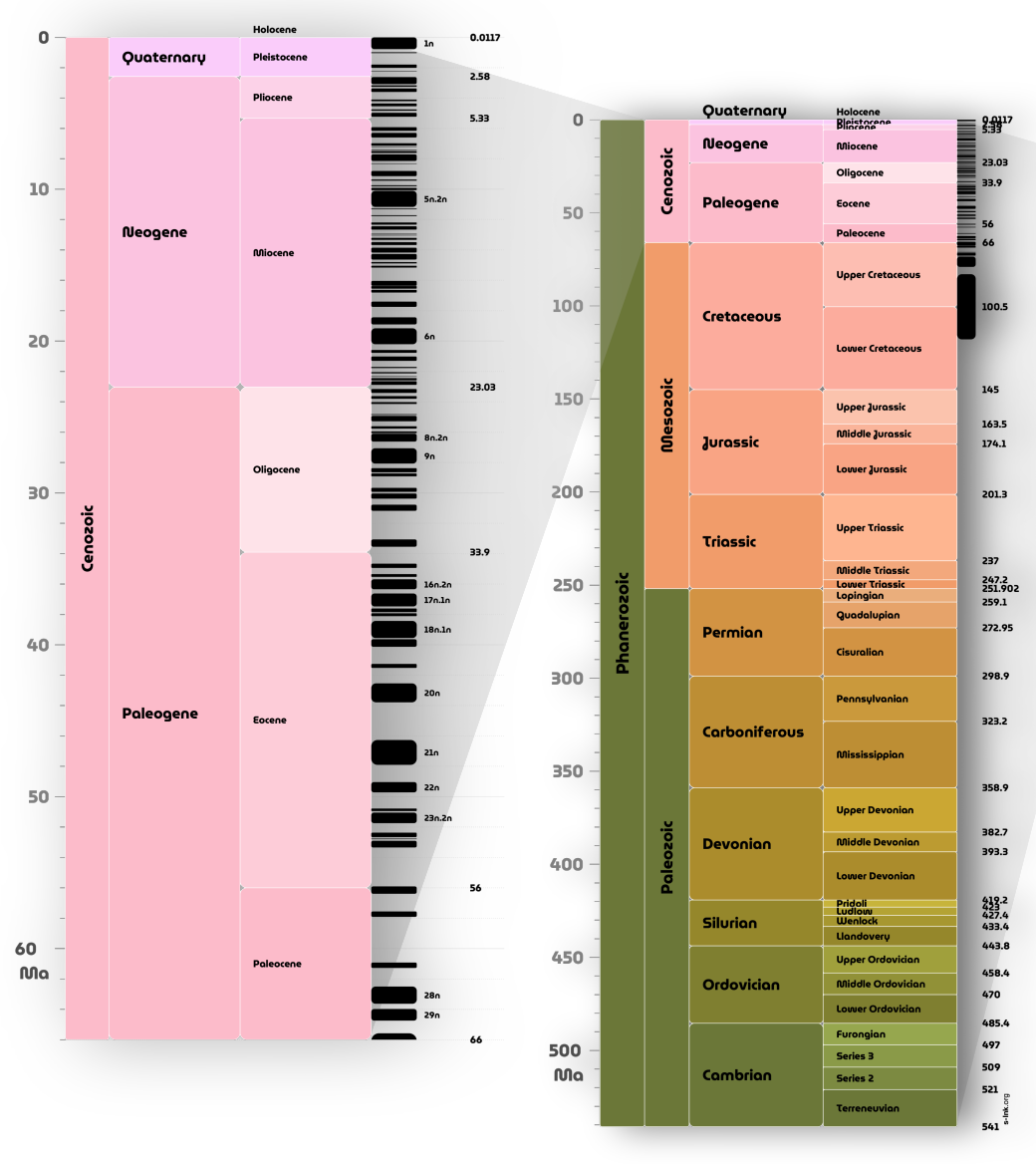

\section{Qeologic Time Scale}

s-Ink.org
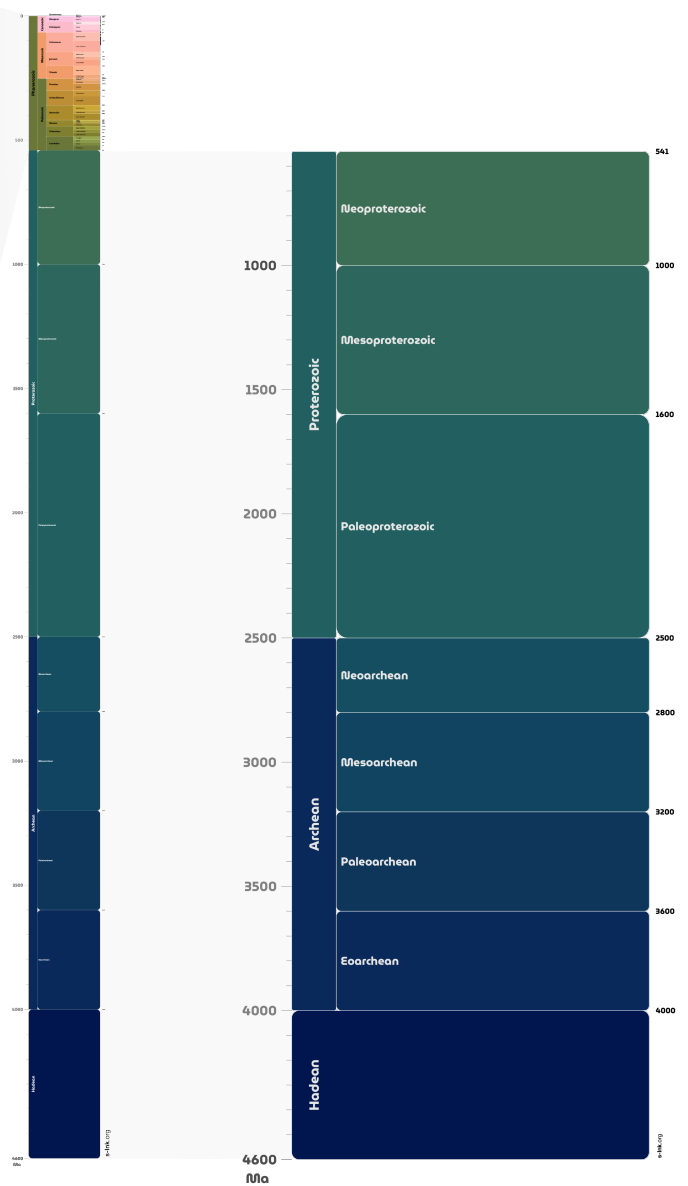

Figure A.7: Time-uniform geologic time scale. As yet another alternative to Figures 3 and A.6, the colour-vision deficiency friendly geologic time scale represents the true relative duration of individual epochs, periods, eras, and eons. Coloured with the categorical scientific batlow colour map from Crameri (2018b). Specimen available at s-ink.org/geologic-time-scale-uniform. 


\section{Bibliography}

Argus, Donald F, Richard G Gordon, and Charles DeMets (20II). "Geologically current motion of 56 plates relative to the no-netrotation reference frame”. In: Geochemistry, Geophysics, Geosystems I2.II.

Bertin, Jacques (1983). Semiology of graphics; diagrams networks maps. Tech. rep. Madison: University of Wisconsin Press.

- (20II). Graphics and graphic information processing. Walter de Gruyter.

Borland, D. and R. M. Taylor Ii (2007). "Rainbow Color Map (Still) Considered Harmful”. In: IEEE Computer Graphics and Applications 27.2, pp. I4-I7. ISSN: 0272-1716.

Brewer, Cynthia A (1994). "Color use guidelines for mapping”. In: Visualization in modern cartography 1994, pp. I23-I48.

Brewer, Cynthia A. (Jan. 1997). "Spectral Schemes: Controversial Color Use on Maps". In: Cartography and Geographic Information Systems 24.4, pp. 203-220. DOI: 10 . 1559 / 152304097782439231. URL: https : / / doi . org / 10 . 1559/152304097782439231.

Crameri, F. (Aug. 2017). The Rainbow Colour Map (repeatedly) considered harmful. edited by G.E. Shephard, EGU GD Blog. URL: http://blogs . egu.eu/divisions/gd/2017/08/ 23/the-rainbow-colour-map/.

- (20I8a). "Geodynamic diagnostics, scientific visualisation and StagLab 3.o". In: Geoscientific Model Development II.6, pp. 254I-2562. DOI: 10 . 5194/gmd-11-2541-2018. URL: https : / / www . geosci-model-dev . net/11/2541/ 2018/.

Crameri, Fabio (20I8b). Scientific colour maps. Zenodo. DoI: 10 . 5281/zenodo . 1243862. URL: https : / /ww . doi . org/ 10.5281 /zenodo. 1243862 .

- (2020). deLight: advanced colour image inversion. Zenodo. DOI: 10.5281/zenodo. 3634422. uRL: https://doi.org/10. $5281 /$ zenodo. 3634422 .

Crameri, Fabio, Grace E. Shephard, and Clinton P. Conrad (2019). "Plate Tectonics*". In: Reference Module in Earth Systems and Environmental Sciences. Elsevier. ISBN: 978-0-I2-409548-9. DOI: https : / / doi .org / 10 . 1016/B978 - 0 - 12 - 4095489.12393-0. URL: http: / / www . sciencedirect.com/ science/article/pii/B9780124095489123930.

Crameri, Fabio, Grace E Shephard, and Philip J Heron (2020). "The misuse of colour in science communication". In: Nature Communications II.I, p. 5444. DOI: 10 . 1038/s41467-02019160 - 7. URL: https : / / doi . org/10 . 1038/s41467 020-19160-7.

Crameri, Fabio et al. (Mar. 2018). "The dynamic life of an oceanic plate”. In: Tectonophysics. ISSN: O040-I95I. DOI: 10 . 1016 / j . tecto . 2018 . 03 . 016. URL: http : / / www . sciencedirect . com/science / article / pii / S0040195118301215.
Crameri, Fabio et al. (2020). "A transdisciplinary and communitydriven database to unravel subduction zone initiation". In: $\mathrm{Na}$ ture Communications II.I, p. 375o. DOI: 10 . 1038 / s41467 020 - 17522 - 9. URL: https : / / doi . org / 10 . 1038 / s41467-020-17522-9.

Dent, Borden D, Jeffrey S Torguson, and Thomas W Hodler (1999). Cartography: Thematic mapdesign. Vol. 5. WCB/McGraw-Hill New York.

Hagh-Shenas, H. et al. (2007). "Weaving Versus Blending: a quantitative assessment of the information carrying capacities of two alternative methods for conveying multivariate data with color." In:IEEE Transactions on Visualization and Computer Graphics I3.6, pp. I270-I277. DOI: 10 .1109/TVCG . 2007.70623.

Hawkins, Ed (Mar. 2015). “Scrap rainbow colour scales”. In: Nature 519, 29I EP -. URL: https : / / doi .org / 10 . 1038 / 519291d.

Illingworth, Sam (2017). "Delivering effective science communication: advice from a professional science communicator”. In: Seminars in Cell छ Developmental Biology 70. Science communication in the field of fundamental biomedical research Biology of aging: new models, new methods, pp. IO-I6. ISSN: I084-952I. DoI: https : / / doi . org/10 .1016/ j . semcdb . 2017 . 04. 002. URL: https : / / wWw . sciencedirect . com / science/article/pii/S1084952117301933.

Kovesi, Peter (20I5). "Good Colour Maps: How to Design Them". In: CoRR abs/I509.03700. URL: http: //arxiv .org/abs / 1509.03700.

Light, Adam and Patrick J. Bartlein (2004). "The end of the rainbow? Color schemes for improved data graphics". In: Eos Trans. $A G U$ 85.40, pp. 385-39I. ISSN: 2324-9250. URL: http: // dx . doi.org/10.1029/2004E0400002.

MacEachren, Alan M (1994). Some truth with maps: A primer on symbolization and design. Assn of Amer Geographers.

- (2004). How maps work: representation, visualization, and design. Guilford Press.

Makri, Anita (2017). "Give the public the tools to trust scientists". In: Nature 541.7637, pp. 26I-26I. DoI: 10 . 1038/541261a. URL: https://doi.org/10.1038/541261a.

Monmonier, Mark (2018). How to lie with maps. University of Chicago Press.

Morrison, Mike (2019). Better Scientific Poster. URL: https : / / osf.io/ef53g/.

Müller, R. Dietmar et al. (Feb. 1997). "Digital isochrons of the world's ocean floor”. In: J. Geophys. Res. IO2.B2, pp. 32II-32I4. ISSN: 2156-2202. URL: http : / / dx . doi . org/10 . 1029/ 96JB01781.

Murchie, Karen J. and Dylan Diomede (2020). "Fundamentals of graphic design-essential tools for effective visual science communication”. In: FACETS 5.I, pp. 409-422. DOI: $10.1139 /$ facets - 2018 - 0049. eprint: https : / / doi o org / 10 . 
1139/facets-2018-0049. uRL: https : //doi .org/10 . 1139/facets-2018-0049.

Robinson, AH (1960). Elements of Cartography. John Wiley and Sons, New York.

Rogowitz, B. E. and L. A. Treinish (Dec. 1998). "Data visualization: the end of the rainbow". In: IEEE Spectrum 35.12, pp. 52-59. ISSN: OoI8-9235. DOI: 10.1109/6.736450.

Rogowitz, Bernice E. and Lloyd A. Treinish (June 1996). "How not to lie with visualization”. In: Comput. Phys. Io.3, pp. 268-273. ISSN: 0894-I866. DOI: 10 . 1063/1 . 4822401. URL: http : //dx.doi.org/10.1063/1.4822401.

Thyng, Kristen M. et al. (2016). "True Colors of Oceanography: Guidelines for Effective and Accurate Colormap Selection”. In: Oceanography 29.3, pp. 9-13. ISSN: 10428275, 2377617X. DOI: 10 . 2307 / 24862699. uRL: http : / / www . jstor . org / stable/24862699.

Torsvik, Trond H. et al. (2016). "Earth evolution and dynamics-a tribute to Kevin Burke". In: Canadian Journal of Earth Sciences 53.II, pp. I073-IO87. DOI: 10.1139/c jes-2015-0228. eprint: https : / /doi .org/10.1139/cjes-2015-0228. URL: https : //doi.org/10.1139/cjes-2015-0228.

Travis, David (199I). Effective color displays: Theory and practice. Vol. 1991. Academic press London.

Tufte, Edward R (1990). Envisioning information. Vol. 126. Graphics press.

Ware, C. (Sept. 1988). "Color sequences for univariate maps: theory, experiments and principles”. In: IEEE Computer Graphics and Applications 8.5, pp. 4I-49. ISSN: 0272-I7I6. DOI: 10.1109/ 38.7760. 